\title{
Cancer risk among professional inshore divers in Norway
}

\author{
Ågot Irgens ${ }^{1}$, Marit Grønning ${ }^{1,2}$ \\ ${ }^{1}$ Norwegian Centre for Diving Medicine, Department of Occupational Medicine, Haukeland University Hospital, Bergen, Norway \\ ${ }^{2}$ Department of Clinical Medicine, University of Bergen, Norway
}

\begin{abstract}
Background: Professional divers are exposed to physiological effects of increased ambient pressure and may be exposed to hazards like carcinogens in polluted breathing gas and water. Consequences in terms of adverse health effects and mortality have been debated for many years.

Aim: The aim of this study was to compare the risk of cancer in Norwegian professional divers to that of the general population.

Materials and methods: All professional divers born 1950-1990 and registered by compulsory notification in The Norwegian Labour Inspection Authority's registry of divers were included and grouped according to the type of diving certificate. By August 2010, 5,526 male divers were identified. The rest of the male population born in the same period $(1,591,243)$ was used as referents. The Cancer Registry of Norway, based on compulsory notification of all diagnosed malignancies, was linked to the Diver Registry.

Results: A higher risk of cancer, OR 1.54 (Cl 1.21-1.96), was observed among fully certified divers compared to the referents. No excess risk was observed for professional divers with limited certificate.

Conclusions: Norwegian fully certified divers had higher risk of cancer compared to the Norwegian male population. Based on small numbers, the higher risks among divers within subgroups of cancer sites should be interpreted with care.
\end{abstract}

(Int Marit Health 2013; 64, 4: 202-206)

Key words: cancer, diving, professional diver, occupational risk

\section{INTRODUCTION}

Professional divers may experience physiological effects of increased ambient pressure and breathing gas mixture different from normal air. They may be exposed to many hazards like polluted water and occupational carcinogens [1-3]. Contamination of the breathing gas with several chemical substances during saturation diving has been observed. Metal exposure from fumes during hyperbaric welding is a potential source of carcinogens [4, 5]. Thus, high cancer rates have been observed in construction and metal workers [6]. As divers do welding in both wet and dry compartments, they may be at excess risk of developing cancer [7].

The higher cancer risks among divers exposed to polluted water, reported in Israel [2], have been questioned due to uncertainty about the exposures levels and possible selection bias [8-11]. The most frequent diagnoses were malignant tumours of the gastrointestinal tract, brain/central nervous system, skin and lung. In a retrospective study of fishermen in Singapore, a high incidence of stomach cancer was found in a subgroup of divers [12].

To our knowledge, these are the only papers reporting cancer risk in divers. Due to this lack of knowledge, we wanted to study the risk of cancer among Norwegian professional divers in comparison with the general male population, based on national registries: The National Population Registry (NPR), the official registry of occupational divers and the Cancer Registry of Norway, all based on compulsory notification.

Ågot Irgens, Norwegian Centre for Diving Medicine, Department of Occupational Medicine, Haukeland University Hospital, Box 1400, N-5021 Bergen, Norway,

tel: +47 55973882, fax: +47 55975137, e-mail: agot.irgens@helse-bergen.no 


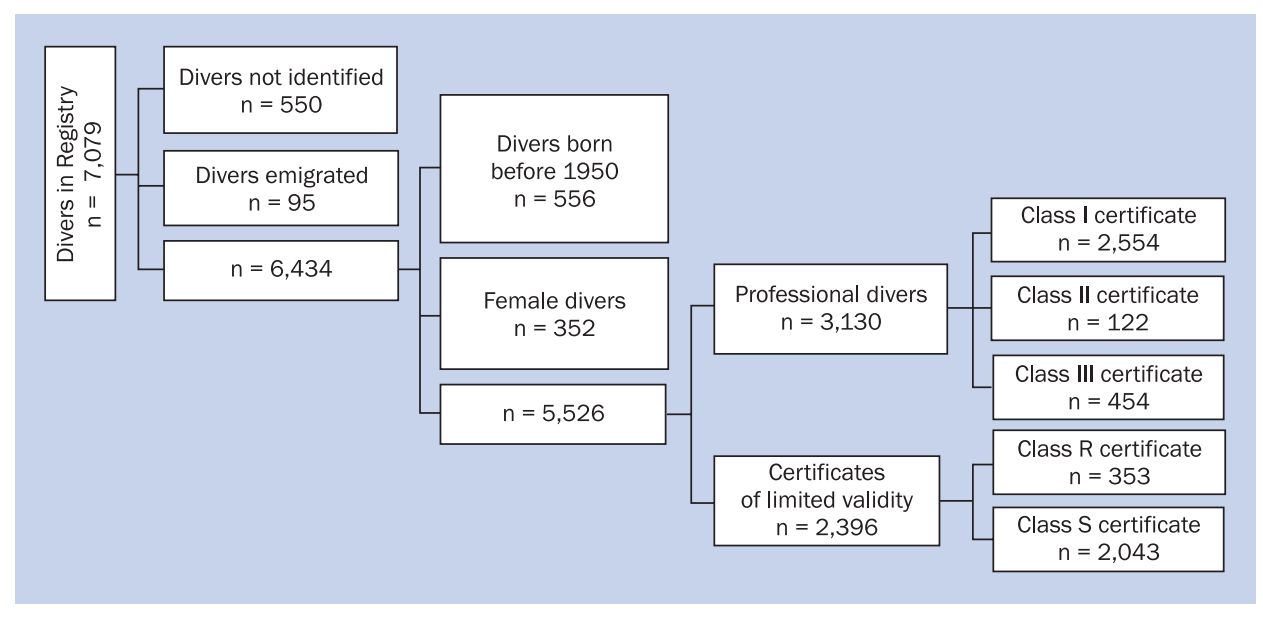

Figure 1. Flow chart showing all divers registered in The Norwegian Inshore Diving Registry by August 2010, and the divers included in the study

\section{MATERIALS AND METHODS}

\section{SUBJECTS}

Norwegian inshore divers are certified by The Norwegian Labour Inspection Authority - a governmental agency on occupational safety and health under the Ministry of Labour. From 1980 onwards, the Labour Inspection Authority has operated the Norwegian Inshore Diver Registry (NIDR), which comprises data on all divers having held a certificate valid for professional inshore diving. By August 2010, 7,079 divers were included in the registry (Fig. 1). For the first years of the registry data are incomplete; consequently, we decided to exclude divers born before 1950. Very few female divers $(n=352)$ were registered, hence women were excluded from the analyses.

The divers were categorised according to the class of certificate. The certificates are graded according to qualification: Class I (HSE part 1 equivalent) requires practical and theoretical training to perform subsea work to a depth of $50 \mathrm{~m}$; Class II (Closed bell) requires the same qualifications as Class I plus theoretical education and practical training in saturation and bell diving; Class III (Standard gear) requires the same qualifications as Class I in addition to practical training and education in specialised subsea work; certificate Classes $R$ and $S$ have limited validity - Class $R$ is restricted to rescue diving to a maximum of $30 \mathrm{~m}$ and Class $\mathrm{S}$ is restricted to specific operations such a scientific diving with SCUBA gear to a maximum of $30 \mathrm{~m}$ and hyperbaric chamber attendance. Divers holding more than one certificate were categorised according to the highest level certification. This is based on exposure risks as ' $I I>I I I>I>R>S$ '.

The divers have been further categorised into 'full certification' (Class II, III and I) and 'limited certification' (Class R and S).

Some divers hold more than one class of certificate, and some have been diving both inshore and offshore. The work typical for each certificate may be associated with various levels of work-related exposure.

The NIDR includes mainly inshore divers. The Petroleum Safety Authority Norway manages a separate registry of offshore divers only. However, data from this registry were not available. Those diving both inshore and offshore are recorded in both registries and were included in the study.

The rest of the other men from the general population born after 1950 were used as referents.

\section{CANCER}

The population-based Cancer Registry of Norway was established in 1951. The notification of all diagnosed malignancies is mandatory. The registry contains data on all incident cancer cases, including date of diagnosis, sex, clinical and morphological type of diagnosis and tumour site. The data quality in the registry is shown to be reasonably accurate, close-to-complete and timely [13]. The cancer diagnoses are categorised according to The International Classification of Diseases (ICD-7 codes).

All cancers and the following cancer subcategories were studied: cancer of buccal cavity and pharynx (140-148), cancer of digestive organs and peritonaeum (150-159), cancer of respiratory system (160-165), cancer of breast and genito-urinary organs (170-181), cancer of other and unspecified sites - including neoplasm of: skin, eye, brain and other parts of nervous system, thyroid gland, other endocrine glands, bone (including jaw bone), connective tissue, secondary and unspecified malignant lymph nodes, other and unspecified sites (190-199), and cancers of lymphatic and haematopoietic tissues (200-207).

Mean age at cancer diagnosis was 40.0 (SD 10.0) among fully certified divers, 35.6 (10.2) among limited certified divers, and 39.5 (10.8) among referents. 
Median age at cancer diagnosis was 39.0 (range 18.0-57.0) among divers and 39.0 (18.0-58.0) among referents. Median age by censoring (end of study) for non-cancer divers was 43.0 (21.0-61.0) and 42.0 (21.0-61.0) among referents.

\section{LINKAGE OF REGISTRIES}

The NPR issues a national identification number (NIN) to all Norwegian citizens. Unfortunately, the Diver Registry does not record the NIN. Based on date of birth, first name and surname, we obtained NIN for each diver from the NPR. Some divers could not be identified (550) and some had emigrated (95). All divers born before 1950 (556) and all female divers (352) were excluded from the analysis. We were able to identify 5,526 male divers born between 1950 and 1990, 3,130 of whom were fully certified professional divers (Fig. 1). The rest of the Norwegian male population born in the same period $(1,591,243)$ served as referents. As professional divers are registered above the age of 18 years, we excluded referents who had died before this age $(13,513)$. All these records were linked to the Cancer Registry. Cancer cases diagnosed before the age of 18 were excluded as well $(2,166)$. None of these cancer patients were divers. The final number of referents used in analysis was $1,575,564$ men.

\section{STATISTICAL ANALYSES}

Cancer risks were compared by odds ratios (OR) obtained by logistic regression analysis, crude and by adjustment for age at diagnosis for cases and age at the end of the study (censored in 2011) for the rest. OR and 95\% confidence intervals $(\mathrm{Cl})$ are presented in Tables 1 and 2 . In the analysis of 'cancer of digestive organs and peritonaeum', only crude OR were calculated from 2 by 2 tables due to few cases. A trend of increasing cancer risk by certificate (None, S, R, I, III, II) was studied by including the type of certificate in the logistic regression analysis as a continuous variable. All statistical analyses were performed by IBM, SPSS Statistics version 21 [14].

The study was approved by the regional committees for medical and health research ethics (2010-01123).

\section{RESULTS}

17.2 per thousand of the divers $(n=95)$ and 15.4 per thousand of the referents had a cancer diagnosis in the Cancer Registry (Table 1). No difference in cancer risk was

Table 1. Cancer among Norwegian professional male divers and referents born 1950 to 1990 by type of certificate*

\begin{tabular}{lllll}
\hline & $\begin{array}{l}\text { Total } \\
\mathbf{n}\end{array}$ & $\begin{array}{l}\text { Cancer } \\
\mathbf{n}(\text { per 1000) }\end{array}$ & Crude odds ratio & Adjusted odds ratio** \\
\hline Referents & $1,575,564$ & $24,251(15.4)$ & 1 & 1 \\
All divers & 5526 & $95(17.2)$ & $1.14(0.93-1.39)$ & $1.17(0.95-1.43)$ \\
S - certificate & 2043 & $25(12.2)$ & $0.81(0.54-1.20)$ & $0.79(0.53-1.17)$ \\
R - certificate & 353 & $2(5.7)$ & $0.37(0.09-1.49)$ & \\
I - certificate & 2554 & $55(21.5)$ & $1.43(1.10-1.87)$ & $1.51(1.15-1.97)$ \\
III - certificate & 454 & $9(19.8)$ & $1.32(0.68-2.55)$ & $1.47(0.76-2.85)$ \\
II - certificate & 122 & $4(32.8)$ & $2.21(0.81-5.97)$ & $2.52(0.93-6.82)$
\end{tabular}

*Deceased before the age of 18 and cancer diagnosis before the age of 18 are excluded

**Adjusted for age at the date of cancer diagnosis for cases and age at the end of the study (2011) for the rest

Table 2. Cancer among Norwegian fully certified male divers and referents born 1950 to $1990 *$

\begin{tabular}{|c|c|c|c|c|}
\hline & $\begin{array}{l}\text { Divers } \\
\text { n (per 1000) }\end{array}$ & $\begin{array}{l}\text { Referents } \\
\text { (per 1000) }\end{array}$ & Crude odds ratio & Adjusted odds ratio** \\
\hline All sites & $68(21.7)$ & $(15.1)$ & $1.45(1.14-1.84)$ & $1.54(1.21-1.96)$ \\
\hline Cancer of buccal cavity and pharynx & $4(1.28)$ & $(0.45)$ & $2.81(1.05-7.52)$ & $2.62(0.98-7.01)$ \\
\hline Cancer of digestive organs and peritonaeum & $1(0.32)$ & $(2.10)$ & $0.15(0.02-1.08)$ & \\
\hline Cancer of respiratory system & $4(1.28)$ & $(1.01)$ & $1.26(0.47-3.36)$ & $1.10(0.41-2.93)$ \\
\hline Cancer of breast and genito-urinary organs & $21(6.71)$ & $(4.97)$ & $1.35(0.88-2.08)$ & $1.53(1.00-2.35)$ \\
\hline Cancer of other and unspecified sites & 25 (7.99) & $(4.62)$ & $1.73(1.17-2.57)$ & $1.97(1.33-2.93)$ \\
\hline $\begin{array}{l}\text { Cancers of lymphatic and haematopoietic } \\
\text { tissues }\end{array}$ & $14(4.47)$ & $(2.28)$ & $1.97(1.16-3.33)$ & $2.31(1.36-3.91)$ \\
\hline
\end{tabular}

*Deaths and cancer diagnosis before the age of 18 are excluded

**Adjusted for age at the date of cancer diagnosis for cases and age at the end of the study (2011) for the rest 
seen between limited certified divers and referents; adjusted OR 0.72 ( $\mathrm{Cl}$ 0.50-1.06).

Cancer risks varied between divers holding different certificates, with the highest risk among fully certified divers (class I/III/II) (Table 1). An increased significant risk was observed among Class I divers only. However, the trend of increasing cancer risk by certificate (None, S, R, I, III, II) was observed ( $p=0.005)$. All cancers and subgroups of cancer among fully certified divers are presented in Table 2. Increased cancer risks (statistical significant) were seen for 'all sites', cancer of lymphatic and haematopoietic tissues and cancer of other and unspecified sites (including melanoma of skin, skin non-melanoma, eye, brain, central nervous system, thyroid, bone, soft tissues). A tendency of increased cancer risk was seen for breast and genito-urinary organs.

\section{DISCUSSION}

Overall, the cancer risk was not increased in the Norwegian cohort of mainly inshore divers compared to the Norwegian general population of males born in the same period.

However, fully certified divers had higher cancer risks than the referents. The highest excess risks were observed for 'cancers of lymphatic and haematopoietic tissues' and for 'cancer of other and unspecified sites' (defined in method). A tendency of increased risk was observed in the category 'cancer of breast and genito-urinary organs'. No increased cancer risk was observed for divers with limited diving certificate.

Based on data from national registries, information or selection biases are not very likely. There are, however, some caveats. The quality of the Diver Registry has never been assessed. Since NIN was not included in this registry, not all divers could be identified due to misspelled or changed names or incorrect dates of birth. Nevertheless, we managed to identify $92 \%$ of the registered divers. Some, but not all, Norwegian offshore divers were included in this study. The Registry comprises only 122 Class II divers, whereas 365 Norwegian divers have been reported to have worked offshore [15] and Class II certificate was required. However, a substantial number of these offshore divers were born before 1950.

The reference population of about 1.6 million men includes up to 50,000 recreational divers in addition to a few compressed air workers, as well as some offshore divers. Thus pressure, hyperoxia, increased gas density etc., are not hazards absolutely unique to the identified diver population, causing conservative estimates. Considering the size of the reference group and the limited number of offshore divers, misclassification of offshore divers as referents should be negligible.

The Diver Registry holds no detailed data on diving activity and exposure, hence possible relationships between carcinogenic factors and cancer risk could not be studied.

Professional divers selected on the basis i.a. of good health would be expected to have cancer risks lower than the general population, provided that no excess occupational hazard exists. This held true, however, only for those divers assumed to be the least exposed (i.e. divers with limited certificates). Thus, those assumed to have the highest diving exposure had higher cancer risk than the general population, possibly due to specific occupational hazards. However, still the risk may be underestimated. The general working population would have been a more suitable reference group in our study. Among occupationally active persons, the cancer incidence is shown to be lower [16], than that for the general population.

To our knowledge, only 1 register-based study has been conducted on cancer among divers [2]. In the paper by Richter et al. [2], the diagnoses were confirmed in the Cancer Registry of Israel and the expected cancer rates were derived from general Jewish male population. The observed-to-expected ratio for all tumours combined was 2.29 $(p<0.01)$. The mean age at diagnosis was 39.5 years, similar to our findings. The Israeli study reported increased risk of malignant tumours of the gastrointestinal tract, brain/ /central nervous system, skin and lung, while we have found increased risk of cancer of other and unspecified sites (defined in method). The frequency of stomach cancer was high also in Chinese divers [12]; this was not confirmed in our study. No increased risk of lung cancer was seen in our study. This might partly be explained by a lower smoking frequency among divers. In 2012 smoking frequency among Norwegian males at similar age was $16 \%$. In an unpublished questionnaire study for this diver population, smoking frequency was $11 \%$.

The Israeli study included only full-time naval divers, who used wet diving suits for diving and had training in a polluted river. The Norwegian divers use dry diving suits and have most likely different exposure both to chemicals and metal fumes. The Class II certificate divers stay in a limited space up to several weeks and might be exposed to potential carcinogens continuously for the whole period. Inshore divers normally do 1 or more dives 4 or 5 days during the week. Expectedly, their exposure time will be shorter.

A review article [5] has summarised the saturation divers' working atmosphere, focusing on benzene. In saturation diving, possible interactions exist of high pressure, elevated partial pressure of oxygen and continuous exposure to chemicals for weeks, which may necessitate special limits to avoid unhealthy chemical exposure [5]. Several divers do welding in both wet and dry compartments involving exposure to benzene and may therefore be at increased risk of developing cancer. Overall, the authors concluded that the evidence regarding the interaction between chemical 
compounds is limited. In addition, hardly any scientific data exist on possible dermal exposures from seawater within the diving suit.

Our study population was rather young and estimates should be interpreted with caution, given the long latency period for most cancer types. This study can therefore not exclude the possibility of stronger effects in older age groups.

Studies focusing on exposure in different categories of divers should be prioritised to be able to improve the risk assessment and thereby establish effective prevention [6].

\section{CONCLUSIONS}

Norwegian fully certified divers had higher risk of cancer compared to the Norwegian male population. The higher risks in groups of cancer sites, however, should be interpreted with care. The results were based on small numbers and the power in the study was low. Further research is needed.

\section{REFERENCES}

1. Bradley ME. Commercial diving fatalities. Aviat Space Environ Med 1984; 55: 721-724.

2. Richter ED, Friedman LS, Tamir $Y$ et al. Cancer risks in naval divers with multiple exposures to carcinogens. Environ Health Perspect 2003; 111: 609-617.

3. Rushton L, Bagga S, Bevan R et al. Occupation and cancer in Britain. Br J Cancer 2010; 102: 1428-1437.

4. Froom P. Determining standards for professional divers diving in benzene polluted waters. Toxicol Ind Health 2008; 24: 525-530.
5. Djurhuus R, Nossum V, Øvrebø S; Skaug V. Proposal on limits for chemical exposure in saturation divers' working atmosphere: the case of benzene. Critical Rev Toxicol 2012; 42: 211-229.

6. Rushton L, Hutchings S, Brown T. The burden of cancer at work: estimation as the first step to prevention. Occup Environ Med 2008; 65: $789-800$

7. t'Mannetje A, Brennan $P$, Zaridze $D$ et al. Welding and lung cancer in Central and Eastern Europe and United Kingdom. Am J Epidemiol 2001; 175: 706-714.

8. Friedman LS, Richter ED. Exposure to water pollution and cancer in Israeli navy divers. Int J Occup Health 2009; 15: 421-423.

9. Amitai $\mathrm{Y}$, Almog S, Herut B. Cancer risk to navy divers questioned. Environ Health Perspect 2003; 111: A630-A631.

10. Friedman LS, Richter ED. Exposure to water pollution and cancer in Israeli Navy divers. Int J Occup Environ Health 2009; 15: 421.

11. Froom $P$. Water pollution and cancer in Israeli navy divers. Int J Occup Environ Health 2009; 15: 326-328.

12. Jeyaratnam J, Lee J, Lee HP, Phoon WO. Stomach cancer incidence in a cohort of fishermen in Singapore. Scand J Work Environ Health 1987; 13: 524-526.

13. Larsen IK, Småstuen M, Johannesen TB et al. Data quality at the Cancer Registry of Norway: an overview of comparability, completeness, validity and timeliness. Eur J Cancer 2009; 45: 1218-1231.

14. IBM Corp. Released 2012. IBM SPSS Statistics for Windows, Version 21.0. Armonk, NY: IBM Corp.

15. NOU. Pionerdykkerne i Nordsjøen. Oslo: Ministry of Government Administration, Reform and Church Affairs, NOU 2003: 5 [Commission of Enquiry to investigate all circumstances related to diving in the North Sea in the pioneer period.].

16. Kirkeleit J, Riise T, Bjørge T, Christiani DC. The healthy worker effect in cancer incidence studies. Am J Epidemiol 2013; 177: 1218-1224. 